\title{
New Generalizations of Caristi's Fixed Point Theorem Via Brézis-Browder Principle
}

\author{
TEMistocle BîRsAN
}

ABstract. In this paper, some generalizations of Caristi's fixed point theorem are obtained via the Brézis-Browder principle: Theorems 2.1, 2.2, 3.1, and 3.2.

\section{InTRODUCTION}

In 1976, J. Caristi [4] has established a fixed point theorem for functions on a complete metric space, and which are not assumed to be continuous. The particular interest for this result derives from its numerous applications in the nonlinear functional analysis as well as from the close relation with the mathematical backgrounds: axiom of choice, and weaker forms of it $([6,7,10]$ etc.). There exists a rich literature concerning the various generalizations of Caristi's fixed point theorem $([1,2,5,6,8,9]$ etc.).

The objective of this paper is to indicate some new extensions of Caristi's fixed point result via Brézis-Browder principle [3].

We state this principle in the form:

Theorem 1.1. Let $(X, \leq)$ be a partial ordered set and let $\theta: X \rightarrow \mathbb{R} \cup\{+\infty\}$ be a function. Suppose that

$1^{\circ}$ every increasing sequence in $X$ is bounded, and

$2^{\circ} \theta$ is an increasing function.

Then, for each $x_{0} \in X$ there exists an element $x^{*} \in X$, with $x_{0} \leq x^{*}$, such that $x^{*} \leq x \Rightarrow \theta(x)=\theta\left(x^{*}\right)$.

We observe that, in order to prove Theorem 1.1, we need to employ only the countable axiom of choice.

\section{FiXED POINTS FOR THE FUNCTIONS}

The following statement includes many generalizations of Caristi's fixed point theorem.

1991 Mathematics Subject Classification. Primary 54H25.

Key words and phrases. fixed point, Caristi's theorem, Brézis-Browder principle, orbit, lower semicontinuous functions. 
Theorem 2.1. Let $(X, d)$ be a complete metric space, $f: X \rightarrow X$ be an arbitrary functions, and $\varphi: X^{k+1} \rightarrow \mathbb{R}$ ( $k$ fixed positive integer) be such that the function

$$
x \rightarrow \varphi\left(x, f x, f^{2} x, \ldots, f^{k} x\right), \quad x \in X,
$$

is lower semicontinuous (l.s.c) and bounded below. If the function $f$ satisfies

$$
d(x, f x) \leq \varphi\left(x, f x, \ldots, f^{k} x\right)-\varphi\left(f x, f^{2} x, \ldots, f^{k+1} x\right), \quad x \in X,
$$

then $f$ has a fixed point.

Proof. The function $\theta: X \rightarrow \mathbb{R}$ defined by $\theta(x)=-\varphi\left(x, f x, \ldots, f^{k} x\right)$ is upper semicontinuous (u.s.c) and bounded above. Then (1) can be writen in the form

$$
d(x, f x) \leq \theta(f x)-\theta(x), \quad x \in X
$$

Also, it is easy to see that the relation $\leq$ defined by

$$
x \leq y \Leftrightarrow \theta(x)+d(x, y) \leq \theta(y)
$$

is a partial order on $X$. By (2), $\theta$ is increasing on $X$ with respect to $\leq$.

Now, let $\left(x_{n}\right)_{n}$ be an increasing sequence in $X$. Then $\left(\theta\left(x_{n}\right)\right)_{n}$ is bounded above and increasing in $\mathbb{R}$. Consequently, $\left(\theta\left(x_{n}\right)\right)_{n}$ is convergent, hence, it is a Cauchy sequence. For $n \leq m$ we have $x_{n} \leq x_{m}$, hence

$$
\theta\left(x_{n}\right)+d\left(x_{n}, x_{m}\right) \leq \theta\left(x_{m}\right)
$$

or

$$
d\left(x_{n}, x_{m}\right) \leq \theta\left(x_{m}\right)-\theta\left(x_{n}\right),
$$

and, consequently, $\left(x_{n}\right)_{n}$ is a Cauchy sequence. The space $X$ being complete, there exists $\xi \in X$ such that $x_{n} \underset{n}{\rightarrow} \xi$. Passing to the limit in (3) as $m \rightarrow \infty$, we deduce

$$
\theta\left(x_{n}\right)+d\left(x_{n}, \xi\right) \leq \lim _{m \rightarrow \infty} \theta\left(x_{m}\right)
$$

Because $\theta$ is l. s. c. we have $\lim _{m \rightarrow \infty} \theta\left(x_{m}\right) \leq \theta(\xi)$. Therefore, we obtain

$$
\theta\left(x_{n}\right)+d\left(x_{n}, \xi\right) \leq \theta(\xi), \quad n \in \mathbb{N},
$$

that is $x_{n} \leq \xi, n \in \mathbb{N}$. Hence, $\left(x_{n}\right)_{n}$ is bounded. Thus, we can apply the BrézisBrowder principle to the space $(X, \leq)$ and the function $\theta$ introduced above: for any $x_{0} \in X$ there exists $x^{*} \in X$, with $x_{0} \leq x^{*}$ such that

$$
x^{*} \leq x \Rightarrow \theta(x)=\theta\left(x^{*}\right) .
$$

We shall prove that $x^{*}$ is a fixed point of $f$. Indeed, by $\left(1^{\prime}\right)$ we have

$$
d\left(x^{*}, f x^{*}\right) \leq \theta\left(f x^{*}\right)-\theta\left(x^{*}\right)
$$

and, taking into account (2), we deduce $x^{*} \leq f x^{*}$. In view of (4), it follows $\theta\left(f x^{*}\right)=\theta\left(x^{*}\right)$ and, as a consequence of $(5)$, we obtain $d\left(x^{*}, f x^{*}\right) \leq 0$. Hence $x^{*}=f x^{*}$.

An easy generalization of the preceding theorem is given by

Theorem 2.2. Let $X, f, \varphi$ be given as in Theorem 2.1. If the function $f$ verifies the following condition: for any $x \in X$ there exists $y \in X$ such that 
(i) $d(x, y) \leq \varphi\left(x, f x, \ldots, f^{k} x\right)-\varphi\left(y, f y, \ldots, f^{k} y\right)$, and

(ii) $d(y, f y) \leq \varphi\left(y, f y, \ldots, f^{k} y\right)-\varphi\left(f x, f^{2} y, \ldots, f^{k+1} y\right)$, then $f$ has a fixed point.

Proof. We define the function $\theta$ and the partial order $\leq$ on $X$ as in the proof of theorem 2.1. Therefore, the relations $(i)$ and (ii) can be rewritten in the form

(j) $d(x, y) \leq \theta(y)-\theta(x)$, or $x \leq y$ (by $(2))$, and

(jj) $d(y, f y) \leq \theta(f y)-\theta(y)$, or $y \leq f y$.

One can similarly prove that an increasing sequence in $X$ is bounded.

Consequently, by Brézis-Browder principle, for each $x_{0} \in X$ there exists $x^{*} \in$ $X$, with $x_{0} \leq x^{*}$, such that the implication (4) holds. We prove that $x^{*}$ is a fixed point of $f$ in an analogous manner as in the proof of theorem 2.1, except for few modifications. Indeed, by our hypotheses, for $x^{*}$ there is $y^{*} \in X$ such that

$$
\begin{aligned}
d\left(x^{*}, y^{*}\right) & \leq \theta\left(y^{*}\right)-\theta\left(x^{*}\right), \text { i.e. } x^{*} \leq y^{*}, \text { and } \\
d\left(y^{*}, f y^{*}\right) & \leq \theta\left(f y^{*}\right)-\theta\left(y^{*}\right), \text { i.e. } y^{*} \leq f y^{*} .
\end{aligned}
$$

By (4), the relations $x^{*} \leq y^{*}$ and $y^{*} \leq f y^{*}$ imply that $\theta\left(x^{*}\right)=\theta\left(y^{*}\right)=\theta\left(f y^{*}\right)$. In view of $(6),(7)$, we obtain $d\left(x^{*}, y^{*}\right) \leq 0$ and $d\left(y^{*}, f y^{*}\right) \leq 0$. Hence $x^{*}=y^{*}=$ $f y^{*}$.

We mention some interesting cases of functions $\varphi: X^{k+1} \rightarrow \mathbb{R}$.

I If $k=0$, then (1) takes the form

$$
d(x, f x) \leq \varphi(x)-\varphi(f x), \quad x \in X,
$$

and theorem 2.1 is just Caristi's fixed point theorem, while theorem 2.2 is Theorem 4.4 in [1].

II If $\varphi\left(x_{0}, x_{1}, \ldots, x_{k}\right)=\psi_{0}\left(x_{0}\right)+\psi_{1}\left(x_{1}\right)+\cdots+\psi_{k}\left(x_{k}\right)$, then the condition (1) becomes

$$
\begin{aligned}
d(x, f x) & \leq\left[\psi_{0}(x)-\psi_{0}(f x)\right]+\left[\psi_{1}(f x)-\psi_{1}\left(f^{2} x\right)\right]+\cdots+ \\
& +\left[\psi_{k}\left(f^{k} x\right)-\psi_{k}\left(f^{k+1} x\right)\right], \quad x \in X .
\end{aligned}
$$

III If $\varphi\left(x_{0}, x_{1}, \ldots, x_{k}\right)=\alpha_{0} \psi\left(x_{0}\right)+\alpha_{1} \psi\left(x_{1}\right)+\cdots+\alpha_{k} \psi\left(x_{k}\right)$ (i. e. in the case II we take $\psi_{0}=\alpha_{0} \psi, \psi_{1}=\alpha_{1} \psi, \ldots, \psi_{k}=\alpha_{k} \psi$, where $\left.\alpha_{0}, \alpha_{1}, \ldots, \alpha_{k} \in \mathbb{R}\right)$, then (1) has the form

$$
\begin{aligned}
d(x, f x) & \leq \alpha_{0} \psi(x)+\left(\alpha_{1}-\alpha_{0}\right) \psi(f x)+\cdots+ \\
& +\left(\alpha_{k}-\alpha_{k-1}\right) \psi\left(f^{k} x\right)-\alpha_{k} \psi\left(f^{k+1} x\right), \quad x \in X .
\end{aligned}
$$

IV If $\varphi\left(x_{0}, x_{1}, \ldots, x_{k}\right)=\psi\left(x_{0}\right)+\psi\left(x_{1}\right)+\cdots+\psi\left(x_{k}\right)$ (i. e. in the case III $\alpha_{0}=\alpha_{1}=\cdots=\alpha_{k}=1$ ), then (1) can be rewritten as

$$
d(x, f x) \leq \psi(x)-\psi\left(f^{k+1} x\right), \quad x \in X .
$$


$\mathbf{V}$ If $\varphi\left(x_{0}, x_{1}, \ldots, x_{k}\right)=\psi_{0}\left(x_{0}\right)+\psi_{1}\left(x_{2}\right)+\cdots+\psi_{k}\left(x_{2 k}\right)$, then the inequality (1) is equivalent to

$$
\begin{aligned}
d(x, f x) & \leq\left[\psi_{0}(x)-\psi_{0}(f x)\right]+\left[\psi_{1}\left(f^{2} x\right)-\psi_{1}\left(f^{3} x\right)\right]+\cdots+ \\
& +\left[\psi_{k}\left(f^{2 k} x\right)-\psi_{k}\left(f^{2 k+1} x\right)\right], \quad x \in X .
\end{aligned}
$$

VI Taking $\psi_{0}=\psi_{1}=\cdots=\psi_{k}=\psi$ in $\mathrm{V}$, we obtain $\varphi\left(x_{0}, x_{1}, \ldots, x_{k}\right)=$ $\psi\left(x_{0}\right)+\psi\left(x_{2}\right)+\cdots+\psi\left(x_{2 k}\right)$, and, therefore

$$
\begin{aligned}
d(x, f x) & \leq \psi(x)-\psi(f x)+\psi\left(f^{2} x\right)-\psi\left(f^{3} x\right)+\cdots+ \\
& +\psi\left(f^{2 k} x\right)-\psi\left(f^{2 k+1} x\right), \quad x \in X
\end{aligned}
$$

This condition is utilized by M. R. Tasković in theorem 1 of [8].

VII Taking $\psi_{0}=\psi_{1}=\cdots=\psi_{k}=-\psi$ in the case $\mathrm{V}$, then we obtain the inequality

$$
d(x, f x) \leq \psi(f x)-\psi(x)+\cdots+\psi\left(f^{2 k+1} x\right)-\psi\left(f^{2 k} x\right), \quad x \in X,
$$

which is also utilized by M. R. Tasković (theorem 1a [9]).

We conclude this section with two remarks concerning the condition of l.s.c. imposed to the function $x \rightarrow \varphi\left(x, f x, \ldots, f^{k} x\right), x \in X$.

Remark 2.1. If $f: X \rightarrow X$ is a continuous and $\varphi: X^{k+1} \rightarrow \mathbb{R}$ is l.s.c., then $x \rightarrow \varphi\left(x, f x \ldots, f^{k} x\right), x \in X$, is l. s. c.

Remark 2.2. If $f: X \rightarrow X$ is continuous and $\psi: X \rightarrow \mathbb{R}$ is l.s.c., then the function $x \rightarrow \psi(x)+\psi\left(f^{2} x\right)+\cdots+\psi\left(f^{2 k} x\right), x \in X$ (see (13) and (14)) is l.s.c.

\section{Fixed POINTS FOR THE MULTifunCtions}

Let $F: X \rightsquigarrow X$ be a multifunction with nonempty values. A point $\xi \in X$ is said to be a fixed point for $F$ if $\xi \in F \xi$. The orbit of $x \in X$, denoted by $O(x, \infty)$, is a sequence $\left(x_{n}\right)_{n \in \mathbb{N}}$ which verify the conditions: $x_{0}=x, x_{n+1} \in F x_{n}, n \in \mathbb{N}$.

Theorem 3.1. Let $(X, d)$ be a complete metric space and let $F: X \rightsquigarrow X$ be a multifunction with nonempty values. Suppose that for each $x \in X$ an orbit $O(x, \infty)$ is given, and $\varphi: X^{k+1} \rightarrow \mathbb{R}$ is a function such that the function $x \rightarrow$ $\varphi\left(x_{0}, x_{1}, \ldots, x_{k}\right), x \in X$, where $x_{0}, x_{1}, \ldots, x_{k} \in O(x, \infty)$, is l.s.c. and bounded below. If for each $x=x_{0} \in X$

$$
d\left(x, x_{1}\right) \leq \varphi\left(x_{0}, x_{1}, \ldots, x_{k}\right)-\varphi\left(x_{1}, x_{2}, \ldots, x_{k+1}\right),
$$

then the multifunction $F$ admits a fixed point.

Proof. Define the function $\theta: X \rightarrow \mathbb{R}$ by $\theta(x)=-\varphi\left(x_{0}, x_{1}, \ldots, x_{k}\right)$, where $x_{0}, x_{1}, \ldots, x_{k} \in O(x, \infty)$. Then, the relation $\leq$ given by $x \leq y \Leftrightarrow \theta(x)+d(x, y) \leq$ $\theta(y)$ is a partial order on $X$. As in theorem 2.1, we can apply the Brézis-Browder principle. Hence, for each $x_{0} \in X$ there is $x^{*} \in X$, with $x_{0} \leq x^{*}$, such that $x^{*} \leq x \Rightarrow \theta(x)=\theta\left(x^{*}\right)$. By $(15)$, we have

$$
d\left(x^{*}, x_{1}^{*}\right) \leq \theta\left(x_{1}^{*}\right)-\theta\left(x^{*}\right),
$$


that is $x^{*} \leq x_{1}^{*}$ (with $x_{1}^{*} \in F x^{*}$ ). Therefore, $\theta\left(x_{1}^{*}\right)=\theta\left(x^{*}\right)$, and, taking into account (16), it follows that $x^{*}=x_{1}^{*}$. Hence $x^{*} \in F x^{*}$, that is $x^{*}$ is a fixed point of $F$.

Remark 3.1. In fact, the proof utilizes only the finite orbits $O(x, k+1):=$ $\left\{x, x_{1}, \ldots, x_{k+1}\right\}$, where $x_{0}=x$ and $x_{i+1} \in F x_{i}, i=\overline{1, k}$.

The next theorem generalizes theorem 3.1 in a similar manner as theorem 2.2 generalizes theorem 2.1 .

Theorem 3.2. Let $X, f, \varphi$ be given as in theorem 3.1. If the multifunction $F$ verifies the condition that for each $x \in X$ there is $y \in X$ such that

$$
\begin{aligned}
d(x, y) & \leq \varphi\left(x_{0}, x_{1}, \ldots, x_{k}\right)-\varphi\left(y_{0}, y_{1}, \ldots, y_{k}\right), \text { and } \\
d\left(y, y_{1}\right) & \leq \varphi\left(y_{0}, y_{1}, \ldots, y_{k}\right)-\varphi\left(y_{1}, y_{2}, \ldots, y_{k+1}\right)
\end{aligned}
$$

where $x_{0}, x_{1}, \ldots, x_{k} \in O(x, \infty)$ and $y_{0}, y_{1}, \ldots, y_{k+1} \in O(y, \infty)$, then $F$ admits a fixed point.

\title{
REFERENCES
}

[1] T. Bîrsan, Applications of Brézis-Browder principle to the existence of fixed points and endpoints for multifunction, Balkan J. of Geometry and Its Applications, 3(1998), 23-32.

[2] A. Bollenbacher, T. L. Hicks: A fixed point theorem revisited, Proc. Amer. Math. Soc., 102(1988), 898-900.

[3] H. Brézis, F. Browder: A general principle on ordered sets in nonlinear functional analysis, Adv. in Math., 21(1976), 355-364.

[4] J. Caristi: Fixed point theorems for mappings satisfying inwardness conditions, Trans. Amer. Math. Soc., 215 (1976), 241-251.

[5] D. Downing, W. A. Kirk: A generalization of Caristi's theorem with applications to nonlinear mapping theory, Pacific J. Math., 69(1977), 339-345.

[6] J. R. Jachymski: Caristi's fixed point theorem and selections of set-valued contractions, J. Math. Anal. Appl., 227 (1998), 55-67.

[7] W. A. Kirk: Caristi's fixed point theorem and metric convexity, Colloq. Math., 36(1976), $81-86$.

[8] M. R. Tasković: A directly extension of Caristi fixed point theorem, Math. Moravica, 1(1997), $105-108$.

[9] M. R. Tasković: New geometric fixed point theorems, Math. Moravica, 2(1998), 143-148.

[10] M. Turinici: Mean value theorems on abstract spaces, Math. Nachr., 115(1984), 21-31.

\author{
TEChNiCAL University \\ "GH. ASACHI" OF IAŞI \\ Department of Mathematics \\ 700506 IAŞI - ROMANIA \\ E-mail address: tbi@math.tuiasi.ro
}

